\title{
DOES INHALED NITRIC OXIDE SUPPRESS ENDOGENOUS NITRIC OXIDE PRODUCTION?
}

\author{
Allan P. Goldman, MRCP, S. G. Haworth, FRCP, and D. J. Macrae, FRCA, London, United Kingdom
}

Inhaled nitric oxide (INO) has emerged as a promising selective pulmonary vasodilator for children with corrected $^{1}$ and uncorrected ${ }^{2}$ congenital heart defects. We and others ${ }^{3}$ have noticed, however, that some patients become extremely dependent on this therapy during the first few days of its administration, so that sudden discontinuation of INO at this point in time causes marked instability. We hypothesize that this effect may be due in part to exogenous INO suppressing or down-regulating endogenous nitrous oxide (NO) production by the pulmonary endothelium. Cyclic guanosine monophosphate (cGMP) is the final messenger of the L-arginine nitric oxide pathway. It was postulated that if INO were suppressing endogenous NO production during its early administration, the cyclic cGMP levels would then progressively fall if the INO was discontinued during this period.

A 2-month-old, $3.8 \mathrm{~kg}$ male infant with supracardiac total anomalous pulmonary venous drainage had recur-

From the Cardiothoracic Unit, Great Ormond Street Hospital for Children, London, United Kingdom.

Received for publication Aug. 4, 1995; accepted for publication Sept. 19, 1995.

J Thorac Cardiovasc Surg 1996;112:541-2

Copyright (C) 1996 by Mosby-Year Book, Inc.

$0022-5223 / 96 \$ 5.00+0 \quad \mathbf{1 2 / 5 4 / 6 9 3 4 6}$ rent pulmonary hypertensive crises during the 5 days after complete surgical correction. During these crises, there was an acute rise in pulmonary arterial pressure to suprasystemic levels; this rise could only be controlled with high-pressure hand-bag ventilation with $100 \%$ oxygen. These crises did not abate with maximal conventional therapy, including mechanical ventilation, alkalinization, sedation and neuromuscular blockade, intravenous vasodilators (nitroglycerine at $7 \mu \mathrm{g} \cdot \mathrm{kg}^{-1} \cdot \mathrm{min}^{-1}$ ), and inotropic support (dopamine at $8 \mu \mathrm{g} \cdot \mathrm{kg}^{-1} \cdot \mathrm{min}^{-1}$ ). Cardiac catheterization at this time revealed good hemodynamics, with no evidence of obstructed pulmonary venous flow (right pulmonary capillary wedge pressure $12 \mathrm{~mm} \mathrm{Hg}$, left pulmonary capillary wedge pressure $12 \mathrm{~mm} \mathrm{Hg}$, left ventricular enddiastolic pressure $13 \mathrm{~mm} \mathrm{Hg}$ ), suggesting that the cause of the pulmonary hypertension was a reactive pulmonary vasculature rather than mechanical obstruction. The patient was given a trial of $20 \mathrm{ppm}$ INO as part of ongoing investigational NO studies at our institution. Blood samples for cGMP levels were withdrawn daily from the indwelling arterial catheter both with and without INO. Daily reverse doseresponse studies were conducted to allow INO to be reduced gradually as clinical improvement progressed. This entailed gradually reducing the dose of INO in 1 to 2 ppm aliquots as clinically tolerated. The blood samples were immediately placed on ice and spun at $3000 \mathrm{rpm}$ at $4^{\circ} \mathrm{C}$ for 10 minutes. The serum was stored at $-70^{\circ} \mathrm{C}$ until cGMP concentrations

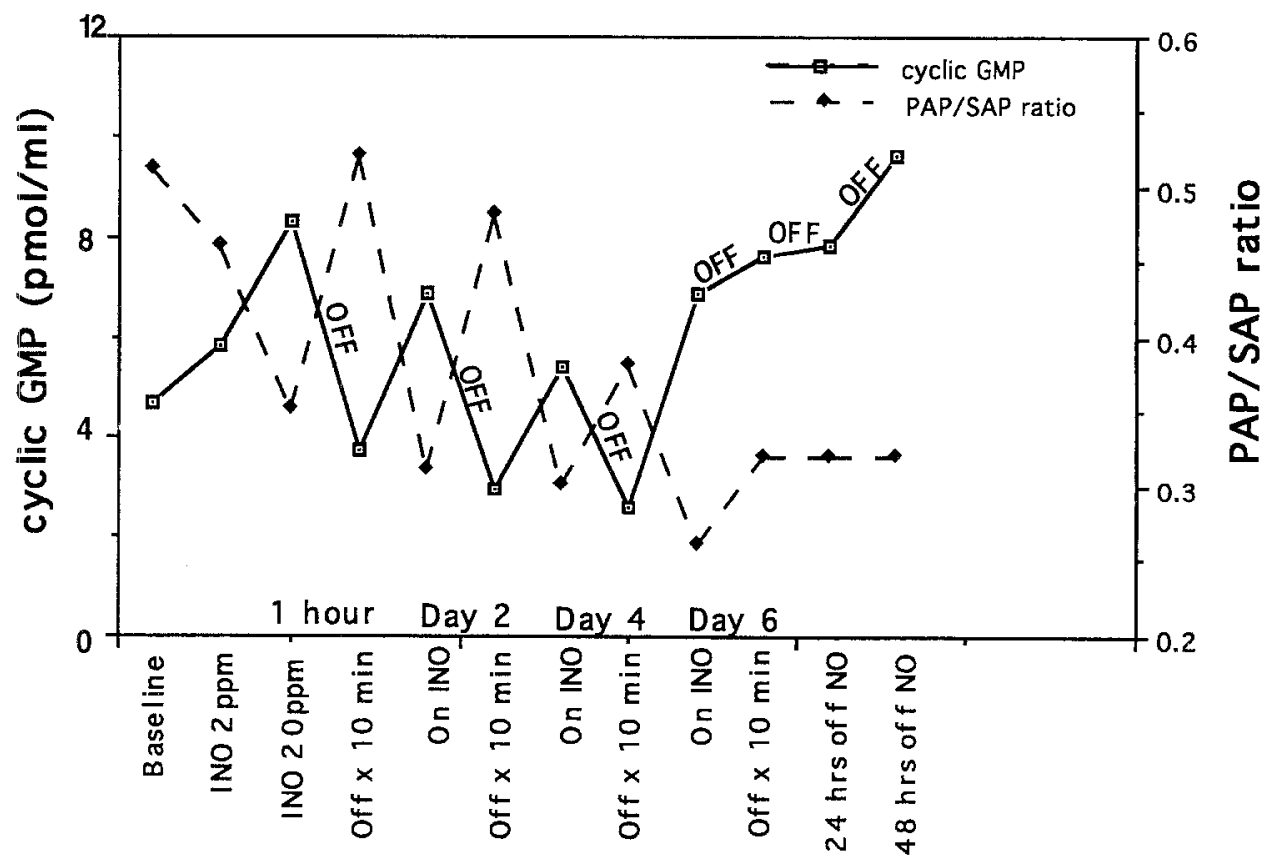

Fig. 1. Concentration of cGMP and ratio of pulmonary arterial pressure $(P A P)$ to systemic arterial pressure $(S A P)$. 
were measured by radioimmunoassay (Amersham RPA525; Amersham International Ltd., Little Chalfont, U.K.). Arterial cGMP concentrations and the mean pulmonary arterialsystemic arterial pressure ratios, recorded with and without INO, are shown in Fig. 1.

Our results show that although the cGMP concentrations increased with the commencement of INO, levels appeared to fall below baseline when attempts were made to wean the patient from INO during the first 6 days of its administration. After 6 days of INO therapy, discontinuation of the INO was no longer associated with a fall in cGMP concentrations; this coincided with clinical resolution of the pulmonary hypertension, suggesting that spontaneous recovery of endogenous $\mathrm{NO}$ production had occurred. The patient was extubated 1 day later and was discharged home after 4 more days.

We believe that these results support the hypothesis that exogenous INO suppresses endogenous NO production after cardiopulmonary bypass when known reversible endothelial damage has occurred. ${ }^{4}$ Exogenous NO has been shown to suppress endogenous NO production in the rat cerebellum by inhibiting nitric oxide synthetase, the rate-limiting enzyme of NO production. ${ }^{5}$ Our findings indicate that similar changes may be occuring in the pulmonary endothelium. An alternative explanation could be that the falling concentrations of cGMP are the result of disease of the pulmonary endothelium, which is ongoing and was itself the initial cause of the pulmonary hypertension.

In conclusion, exogenous INO may suppress endogenous NO production by the pulmonary endothelium during the early course of its administration. This has a number of important clinical implications. First, regular reverse dose-response studies should be conducted to allow the patient to be gradually weaned from INO as clinical improvement and endothelial recovery progress, because rebound pulmonary hypertension may occur if INO treatment is discontinued abruptly. Second, patients already receiving INO who require transfer to tertiary centers may require the INO administration to be continued during the transport. Finally, although prophylactic INO administration may be considered in patients at high risk, its administration may lengthen the time required for mechanical ventilation.

\section{REFERENCES}

1. Miller OI, Celemajer DS, Deanfield JE, Macrae DJ. Very-lowdose inhaled nitric oxide: a selective pulmonary vasodilator after operations for congenital heart disease. J Thorac Cardiovasc Surg 1994;108:487-94.

2. Roberts JD, Lang P, Bigatello LM, Vlahakes GJ, Zapol WM. Inhaled nitric oxide in congenital heart disease. Circulation 1993;87:447-53.

3. Petros AJ. Down-regulation of endogenous nitric oxide production after prolonged administration [Letter]. Lancet 1994; 344:191.

4. Wessel DL, Adatia I, Giglia TM, Thompson JE, Kulik TJ. Use of inhaled nitric oxide and acetylcholine in the evaluation of pulmonary hypertension and endothelial function after cardiopulmonary bypass. Circulation 1993;88(Pt 1):2128-38.

5. Griscavage JM, Fukuto JM, Komori Y, Ignarro LJ. Nitric oxide inhibits neuronal nitric oxide synthase by interacting with the heme prosthetic group. J Biol Chem 1994;269:34: 21644-9.

\section{EVANS BLUE AND GENTIAN VIOLET: ALTERNATIVES TO METHYLENE BLUE AS A SURGICAL MARKER DYE}

Kanili Shoemaker, ${ }^{\mathrm{a}}$ Joseph Rubin, MD, ${ }^{\mathrm{b}}$ G. Lionel Zumbro, $\mathrm{MD},{ }^{\mathrm{c}}$ and Randall Tackett, PhD, ${ }^{\mathrm{a}}$ Athens and Augusta, Ga.

Dyes, such as methylene blue, are used during vascular surgery to aid in the visualization and orientation of the blood vessel. Although methylene blue dye is believed to

From the University of Georgia, College of Pharmacy, Department of Pharmacology and Toxicology, Athens, ${ }^{2}$ the Medical College of Georgia, Department of Thoracic and Cardiac Surgery, Augusta, ${ }^{b}$ and the University Hospital, Department of Surgery, Augusta, Ga. ${ }^{\circ}$

This work was supported by a grant-in-aid from the American Heart Association/Georgia Affiliate.

Received for publication July 28, 1995; accepted for publication Dec. 19, 1995.

Address for reprints: Randall L. Tackett, $\mathrm{PhD}$, Department of Pharmacology and Toxicology, College of Pharmacy, University of Georgia, Athens, GA 30602-2356.

J Thorac Cardiovasc Surg 1996;112:542-4 be innocuous, $\mathrm{w}^{1}$ recently demonstrated that extravascular application of $1 \%$ methylene blue impairs both endothelium-dependent and endothelium-independent vasorelaxation. The present study investigated Evans blue and gentian violet as alternative surgical marker dyes to methylene blue.

Human saphenous veins were cut into 2 to $4 \mathrm{~mm}$ rings, carefully swabbed with $0.5 \%$ Evans blue, $1.0 \%$ gentian violet, or Krebs buffer, and incubated in $20 \mathrm{ml}$ of buffer at $25^{\circ} \mathrm{C}$ for 45 minutes. The vascular rings were then suspended in $10 \mathrm{ml}$ tissue baths containing Krebs buffer, and dose-response curves to acetylcholine, isoproterenol, ve rapamil, and sodium nitroprusside were performed as previously described. ${ }^{1}$

Vascular rings were assayed in duplicate and averaged

Copyright 101996 by Mosby-Year Book, Inc.

$0022-5223 / 96 \$ 5.00+0 \quad \mathbf{1 2 / 5 4 / 7 1 2 6 1}$ 